\title{
ANOSOV DIFFEOMORPHISMS ON A CLASS OF 2-STEP NILMANIFOLDS
}

\author{
KAREL DEKIMPE and SANDRA DESCHAMPS \\ Katholieke Universiteit Leuven, Campus Kortrijk B-8500 Kortrijk, Belgium \\ e-mail:karel.dekimpe@kulak.ac.be
}

(Received 13 January, 2002; accepted 16 September, 2002)

\begin{abstract}
In this paper we study nilmanifolds which are modeled on a quotient of a free 2-step nilpotent Lie group by a 1-dimensional subgroup. In fact we obtain a very easy criterion to decide whether or not such a nilmanifold admits an Anosov diffeomorphism.
\end{abstract}

2000 Mathematics Subject Classification. Primary 37D20; Secondary 20F18, $17 \mathrm{~B} 30$.

1. Anosov diffeomorphisms on nilmanifolds. By a nilmanifold we shall always mean a quotient space $M$ of the form $M=N \backslash G$, where $G$ is a connected and simply connected nilpotent Lie group and $N$ is a lattice of $G$ (i.e. a discrete and cocompact subgroup of $G$ ). If $G$ is a $c$-step nilpotent Lie group, we say that $M$ is a $c$-step nilmanifold and that $M$ is modeled on $G$. Note that $N$ is the fundamental group of $M$.

It is known that any such $N$ is a torsion free, finitely generated nilpotent group and conversely, for any such torsion free, finitely generated group $N$, there is exactly one connected and simply connected nilpotent Lie group $G$ containing $N$ as a lattice. This $G$ is often referred to as the Malcev completion of $N$ ([9], [5]). Let $\mathfrak{g}$ denote the Lie algebra of $G$. Then any automorphism $\varphi$ of $N$ has a unique lift to a continuous automorphism $\tilde{\varphi}$ of $G$. Moreover, $\operatorname{Aut}(G) \cong \operatorname{Aut}(\mathfrak{g})$, where the isomorphism is given by taking the differential of the automorphism (this means that $\tilde{\varphi} \in \operatorname{Aut}(G)$ is identified with $d \tilde{\varphi} \in \operatorname{Aut}(\mathfrak{g}))$.

Definition 1.1. Let $K$ be a subfield of $\mathbb{C}$ and let $\mathfrak{g}$ be a Lie algebra over $K$. An automorphism $\psi \in \operatorname{Aut}(\mathfrak{g})$ is said to be hyperbolic if all of its eigenvalues are of modulus different from 1 .

More generally, we shall say that $\varphi$ or $\tilde{\varphi}$ as above are hyperbolic if the corresponding automorphism $d \tilde{\varphi}$ is hyperbolic.

Any automorphism $\tilde{\varphi}$ of $G$ arising from an automorphism $\varphi$ of $N$ induces a selfdiffeomorphism of the nilmanifold $N \backslash G$. If moreover, $\varphi$ is hyperbolic, we refer to such a self-diffeomorphism as a hyperbolic nilmanifold automorphism. These hyperbolic nilmanifold automorphisms are standard examples of Anosov diffeomorphisms. Let us recall the definition of an Anosov diffeomorphism.

Definition 1.2. A diffeomorphism $f: M \rightarrow M$ on a smooth manifold $M$ is said to be Anosov if there is a continuous splitting of the tangent bundle $T M=E^{s} \oplus E^{u}$ with $d f\left(E^{s}\right)=E^{s}$ and $d f\left(E^{u}\right)=E^{u}$ and there exist constants $C>0$ and $\lambda>1$ such that 
for all positive integers $m$ we have

$$
\left\|(d f)^{m}(v)\right\| \geq C \lambda^{m}\|v\|, \quad \text { if } v \in E^{u} \text { and }\left\|(d f)^{m}(v)\right\| \leq C^{-1} \lambda^{-m}\|v\|, \quad \text { if } v \in E^{s} .
$$

Hence if $f$ is Anosov, one can split the tangent bundle into a contracting and an expanding part.

In [7], A. Manning proved that any Anosov diffeomorphism on a nilmanifold is topologically conjugated to a hyperbolic nilmanifold automorphism. Therefore, the geometric question of which nilmanifolds admit an Anosov diffeomorphism is translated into the group theoretic question of which finitely generated torsion free nilpotent groups admit a hyperbolic automorphism.

This group theoretic question can in turn be translated into a linear algebra problem concerning a rational Lie algebra. To obtain this link, we consider the group $N_{\mathbb{Q}}$, which is the divisible closure (or rational Malcev completion) of $N$. This is the unique divisible group (in which each element has a unique $k$-th root for any positive integer $k$ ) containing $N$ and such that for any element $n \in N_{\mathbb{Q}}$, there exists a positive integer $k$ such that $n^{k} \in N$. (The reader can consult [10] for details on this matter.) Analogously, as for the (real) Malcev completion, we can identify the group $N_{\mathbb{Q}}$ (via exp and $\log$ ) with a Lie algebra $\mathfrak{g}_{\mathbb{Q}}$ over the rationals.

The following translation of the original problem was obtained in [3, Corollary 3.5].

$N$ admits a hyperbolic automorphism if and only if $\mathfrak{g}_{\mathbb{Q}}$ admits a hyperbolic automorphism, for which the corresponding characteristic polynomial has integer coefficients and unit constant term.

Although we have this nice result and the possibility of studying Anosov diffeomorphisms on several mathematical levels, very little is known about the nature of the class of nilmanifolds (nilpotent groups) admitting an Anosov diffeomorphism (hyperbolic automorphism).

Indeed, the problem is solved completely for free abelian groups ([8]) and for finitely generated nilpotent groups of Hirsch length at most 6 ([1], [6]). It is therefore natural to study the situation for 2-step nilmanifolds (2-step nilpotent groups) first.

DEFINITION 1.3. A torsion free, finitely generated, 2-step nilpotent group $N$ is said to be a $T(n, k)$ group if $h\left(\frac{N}{[N, N]}\right)=n$ and $h([N, N])=k$. (Here $h(G)$ denotes the Hirsch length of a group $G$.) Analogously, a 2-step nilpotent Lie algebra $\mathfrak{g}$ over a field $K$ is a $T(n, k)$ Lie algebra if $\operatorname{dim}_{K}\left(\frac{\mathfrak{g}}{[\mathfrak{g}, \mathfrak{g}]}\right)=n$ and $\operatorname{dim}_{K}([\mathfrak{g}, \mathfrak{g}])=k$.

Let $N$ be a torsion free, finitely generated, 2-step nilpotent group, with corresponding rational Lie algebra $\mathfrak{g}_{\mathbb{Q}}$ and corresponding real Lie algebra $\mathfrak{g}$. Then

$$
\begin{aligned}
N \text { is a } T(n, k) \text { group } & \Leftrightarrow \mathfrak{g}_{\mathbb{Q}} \text { is a } T(n, k) \text { Lie algebra } \\
& \Leftrightarrow \mathfrak{g} \text { is a } T(n, k) \text { Lie algebra. }
\end{aligned}
$$

Moreover, we always have that $k \leq \frac{n(n-1)}{2}$.

Concerning hyperbolic automorphisms, the following properties are known.

1. No $T(n, 1)$ group admits a hyperbolic automorphism.

2. A $T\left(n, \frac{n(n-1)}{2}\right)$ group admits a hyperbolic automorphism if and only if $n>2$ ([4]).

3. The situation for $T(n, 2)$ groups is rather complicated, as is already seen in the case of $T(4,2)$ groups ([1], [6]).

These examples indicate that the following reasonable class to investigate is the class of $T\left(n, \frac{n(n-1)}{2}-1\right)=T\left(n, \frac{(n+1)(n-2)}{2}\right)$ groups. 
In this paper we completely solve this case for arbitrary $n$, and find a very easy condition to determine whether or not a $T\left(n, \frac{(n+1)(n-2)}{2}\right)$ group admits a hyperbolic automorphism.

2. Classification of the $T\left(n, \frac{(n+1)(n-2)}{2}\right)$ Lie algebras. As the problem of hyperbolic automorphisms on $T\left(n, \frac{(n+1)(n-2)}{2}\right)$ groups is actually a problem for the corresponding Lie algebras, we start by studying these Lie algebras. The following notion will be used frequently in the rest of the paper.

DEFINITION 2.1. Let $\mathfrak{g}$ be a free 2-step nilpotent Lie algebra over a field $K$ and let $0 \neq X \in[\mathfrak{g}, \mathfrak{g}]$. We define the length of $X$ by

$$
\operatorname{length}(X)=\min \left\{\begin{array}{l|l}
k \in \mathbb{N}_{0} & \begin{array}{l}
X=\left[V_{1}, W_{1}\right]+\ldots+\left[V_{k}, W_{k}\right] \\
\text { for some } V_{1}, V_{2}, \ldots, V_{k}, W_{1}, W_{2}, \ldots, W_{k} \in \mathfrak{g}
\end{array}
\end{array}\right\}
$$

Note that for any automorphism $\varphi$ of $\mathfrak{g}$, we have that

$$
\forall 0 \neq X \in[\mathfrak{g}, \mathfrak{g}]: \operatorname{length}(X)=\operatorname{length}(\varphi(X))
$$

LEMMA 2.2. Let $\mathfrak{g}$ be a free 2-step nilpotent Lie algebra over a field $K$ and let

$$
0 \neq X=\sum_{i=1}^{k}\left[V_{i}, W_{i}\right] \text { for some } V_{i}, W_{i} \in \mathfrak{g}
$$

Let $p: \mathfrak{g} \rightarrow \frac{\mathfrak{g}}{[\mathfrak{g}, \mathfrak{g}]}$ denote the natural projection. If length $(X)=k$, then $\left\{p\left(V_{1}\right), p\left(V_{2}\right)\right.$, $\left.\ldots, p\left(W_{k}\right)\right\}$ is a set of linearly independent vectors.

Proof. Let $k=1$ and assume that $\left\{p\left(V_{1}\right), p\left(W_{1}\right)\right\}$ is linearly dependent. Without loss of generality, we may assume that $p\left(W_{1}\right)=k p\left(V_{1}\right)$, for some $k \in K$, or $W_{1}=$ $k V_{1}+Y$, for some $Y \in[\mathfrak{g}, \mathfrak{g}]$. But then

$$
X=\left[V_{1}, W_{1}\right]=\left[V_{1}, k V_{1}+Y\right]=k\left[V_{1}, V_{1}\right]=0,
$$

which is a contradiction.

Now assume that $k>1$ and suppose that $\left\{p\left(V_{1}\right), p\left(V_{2}\right), \ldots, p\left(W_{k}\right)\right\}$ is not linearly independent. Again without loss of generality we may assume that

$$
W_{k}=\sum_{i=1}^{k} \alpha_{i} V_{i}+\sum_{i=1}^{k-1} \beta_{i} W_{i}+Y, \text { for some } Y \in[\mathfrak{g}, \mathfrak{g}] \text { and some } \alpha_{i}, \beta_{i} \in K .
$$

It follows that

$$
\left[V_{k}, W_{k}\right]=\sum_{i=1}^{k-1}\left(\left[V_{k}, \alpha_{i} V_{i}\right]+\left[V_{k}, \beta_{i} W_{i}\right]\right)
$$


and thus

$$
X=\sum_{i=1}^{k-1}\left(\left[V_{i}, W_{i}\right]+\left[V_{k}, \alpha_{i} V_{i}\right]+\left[V_{k}, \beta_{i} W_{i}\right]\right)
$$

Now we fix an $i$ (between 1 and $k-1$ ) and consider the term

$$
\left[V_{i}, W_{i}\right]+\left[V_{k}, \alpha_{i} V_{i}\right]+\left[V_{k}, \beta_{i} W_{i}\right]
$$

If $\alpha_{i}=\beta_{i}=0$, this sum actually consists of one Lie bracket.

If $\alpha_{i} \neq 0$, this sum equals

$$
\left[V_{k}-\frac{W_{i}}{\alpha_{i}}, \alpha_{i} V_{i}+\beta_{i} W_{i}\right]
$$

Also if $\beta_{i} \neq 0$, this sum equals

$$
\left[V_{k}+\frac{V_{i}}{\beta_{i}}, \alpha_{i} V_{i}+\beta_{i} W_{i}\right]
$$

showing that in every case the term (2) can be written as a single Lie bracket.

Now (1) implies that length $(X) \leq k-1$ which is a contradiction.

COROLlaRY 2.3. Let $\mathfrak{g}$ be a free 2-step nilpotent Lie algebra on $n$ generators. Then for any $X \in[\mathfrak{g}, \mathfrak{g}]$, we have

$$
\operatorname{length}(X) \leq\left\lfloor\frac{n}{2}\right\rfloor
$$

Any $T\left(n, \frac{(n+1)(n-2)}{2}\right)$ Lie algebra is isomorphic to a quotient $\frac{\mathfrak{g}}{\mathfrak{h}}$, where $\mathfrak{g}$ is the free 2-step nilpotent Lie algebra on $n$ generators and $h$ is a 1-dimensional subspace of $[\mathfrak{g}, \mathfrak{g}]$.

Conversely, if $0 \neq X \in[\mathfrak{g}, \mathfrak{g}]$, then $\langle X\rangle$ (the vector space spanned by $X$ ) is a 1dimensional ideal of $\mathfrak{g}$ and $\frac{\mathfrak{g}}{\langle X\rangle}$ is a $T\left(n, \frac{(n+1)(n-2)}{2}\right)$ Lie algebra. In the next theorem we show that the isomorphism type of such a Lie algebra is completely determined by length $(X)$.

TheOREM 2.4. Let $0 \neq X, Y \in[\mathfrak{g}, \mathfrak{g}]$, where $\mathfrak{g}$ is a free 2-step nilpotent Lie algebra on $n$ generators over a field $K$. Then

$$
\frac{\mathfrak{g}}{\langle X\rangle} \cong \frac{\mathfrak{g}}{\langle Y\rangle} \Longleftrightarrow \text { length }(X)=\text { length }(Y)
$$

Proof. There exists an isomorphism

$$
\varphi: \frac{\mathfrak{g}}{\langle X\rangle} \rightarrow \frac{\mathfrak{g}}{\langle Y\rangle}
$$

if and only if there exists an automorphism

$$
\tilde{\varphi}: \mathfrak{g} \rightarrow \mathfrak{g} \text { with } \tilde{\varphi}(\langle X\rangle)=\langle Y\rangle .
$$


Assume that $\tilde{\varphi}$ exists; then $\tilde{\varphi}(X)=k Y$ for some $k \in K \backslash\{0\}$ and so

$$
\text { length }(X)=\text { length }(\tilde{\varphi}(X))=\operatorname{length}(k Y)=\operatorname{length}(Y) .
$$

Now, assume that length $(X)=\operatorname{length}(Y)=k \in \mathbb{N}_{0}$. Then, there exist vectors $V_{1}, V_{2}, \ldots, V_{2 k}, W_{1}, W_{2}, \ldots, W_{2 k} \in \mathfrak{g}$ with

$$
X=\left[V_{1}, V_{2}\right]+\left[V_{3}, V_{4}\right]+\ldots+\left[V_{2 k-1}, V_{2 k}\right]
$$

and

$$
Y=\left[W_{1}, W_{2}\right]+\left[W_{3}, W_{4}\right]+\ldots+\left[W_{2 k-1}, W_{2 k}\right]
$$

By Lemma 2.2, we can choose vectors $V_{2 k+1}, V_{2 k+2}, \ldots, V_{n}, W_{2 k+1}, W_{2 k+2}, \ldots, W_{n} \in$ $\mathfrak{g}$ such that both $V_{1}+[\mathfrak{g}, \mathfrak{g}], V_{2}+[\mathfrak{g}, \mathfrak{g}], \ldots, V_{n}+[\mathfrak{g}, \mathfrak{g}]$ and $W_{1}+[\mathfrak{g}, \mathfrak{g}], W_{2}+$ $[\mathfrak{g}, \mathfrak{g}], \ldots, W_{n}+[\mathfrak{g}, \mathfrak{g}]$ are bases of $\frac{\mathfrak{g}}{[\mathfrak{g}, \mathfrak{g}]}$. As $\mathfrak{g}$ is free 2-step nilpotent on $n$ generators, there is a unique automorphism $\tilde{\varphi}$ of $\mathfrak{g}$ such that

$$
\tilde{\varphi}\left(V_{i}\right)=W_{i} \text { for } i=1,2, \ldots, n .
$$

It is obvious that $\tilde{\varphi}(X)=\tilde{\varphi}(Y)$, which finishes the proof.

Now, we prove the converse of Lemma 2.2.

LEMMA 2.5. Let $\mathfrak{g}$ be a free 2-step nilpotent Lie algebra on $n$ generators over a field $K$ and denote by $p: \mathfrak{g} \rightarrow \frac{\mathfrak{g}}{[\mathfrak{g}, \mathfrak{g}]}$ the natural projection. If $V_{1}, V_{2}, \ldots, V_{k}$, $W_{1}, W_{2}, \ldots, W_{k} \in \mathfrak{g}$ are such that $p\left(V_{1}\right), p\left(V_{2}\right), \ldots, p\left(V_{k}\right), p\left(W_{1}\right), p\left(W_{2}\right), \ldots, p\left(W_{k}\right)$ are linearly independent vectors of $\frac{\mathfrak{g}}{[\mathfrak{g}, \mathfrak{g}]}$, then

$$
X=\left[V_{1}, W_{1}\right]+\left[V_{2}, W_{2}\right]+\ldots+\left[V_{k}, W_{k}\right]
$$

is an element of length $k$ in $[\mathfrak{g}, \mathfrak{g}]$.

Proof. We proceed by induction on $k$ to show that $X$ cannot be written as a sum of fewer Lie brackets. The lemma is obvious for $k=1$.

Let $k>1$. Suppose that the lemma is valid for smaller values of $k$. Assume that length $(X) \neq k$. Then there exist vectors $\tilde{V}_{1}, \tilde{V}_{2}, \ldots, \tilde{V}_{k-1}, \tilde{W}_{1}, \tilde{W}_{2}, \ldots$, $\tilde{W}_{k-1}$, with $\tilde{W}_{k-1} \neq 0$, such that

$$
X=\left[\tilde{V}_{1}, \tilde{W}_{1}\right]+\ldots+\left[\tilde{V}_{k-1}, \tilde{W}_{k-1}\right]
$$

Note that it is possible to choose all the vectors $\tilde{V}_{i}, \tilde{W}_{i}$ in the vector space spanned by $V_{1}, V_{2}, \ldots, V_{k}, W_{1}, W_{2}, \ldots, W_{k}$. Let $I$ be the ideal of $\mathfrak{g}$ generated by $\tilde{W}_{k-1}$ and let $q$ denote the natural projection of $\mathfrak{g}$ onto $\frac{\mathfrak{g}}{[\mathfrak{g}, \mathfrak{g}]+I}$. Then $q\left(V_{1}\right), q\left(V_{2}\right), \ldots, q\left(V_{k}\right)$, $q\left(W_{1}\right), q\left(W_{2}\right), \ldots, q\left(W_{k}\right)$ form a linearly dependent set of vectors $\left(q\left(\tilde{W}_{k-1}\right)=0\right)$ generating a $(2 k-1)$-dimensional subspace of $\frac{\mathfrak{g}}{[\mathfrak{g}, \mathfrak{g}]+I}$. (Use the fact that $\operatorname{dim}\left(\frac{\mathfrak{g}}{[\mathfrak{g}, \mathfrak{g}]}\right)=$ $\left.\operatorname{dim}\left(\frac{\mathfrak{g}}{[\mathfrak{g}, \mathfrak{g}]+I}\right)+1.\right)$

We can assume that $q\left(W_{k}\right)$ is a linear combination of $q\left(V_{1}\right), \ldots, q\left(V_{k}\right)$, $q\left(W_{1}\right), \ldots, q\left(W_{k-1}\right)$ and that the vectors $q\left(V_{1}\right), \ldots, q\left(V_{k}\right), q\left(W_{1}\right), \ldots, q\left(W_{k-1}\right)$ are linearly independent. It follows that

$$
\begin{aligned}
{\left[V_{1}, W_{1}\right]+\ldots+\left[V_{k}, W_{k}\right]+I } & =\left[\tilde{V}_{1}, \tilde{W}_{1}\right]+\ldots+\left[\tilde{V}_{k-1}, \tilde{W}_{k-1}\right]+I \\
& =\left[\tilde{V}_{1}, \tilde{W}_{1}\right]+\ldots+\left[\tilde{V}_{k-2}, \tilde{W}_{k-2}\right]+I .
\end{aligned}
$$


In the proof of Lemma 2.2 we showed that (in case $q\left(W_{k}\right)$ is written as a linear combination of the $q\left(V_{i}\right)$ and the other $q\left(W_{i}\right)$, and the Lie algebra we are working in is $\left.\frac{\mathfrak{g}}{I}\right)$, how to rewrite the expression $\left[V_{1}, W_{1}\right]+\ldots+\left[V_{k}, W_{k}\right]+I$ as a sum of $k-1$ Lie brackets

$$
\left[V_{1}^{\prime}, W_{1}^{\prime}\right]+\ldots+\left[V_{k-1}^{\prime}, W_{k-1}^{\prime}\right]+I .
$$

The reader can check that (during and) after this rewriting process, the elements obtained $q\left(V_{1}^{\prime}\right), \ldots, q\left(V_{k-1}^{\prime}\right), q\left(W_{1}^{\prime}\right), \ldots, q\left(W_{k-1}^{\prime}\right)$ are linearly independent (because we started from a linear independent set $\left.q\left(V_{1}\right), \ldots, q\left(V_{k}\right), q\left(W_{1}\right), \ldots, q\left(W_{k-1}\right)\right)$.

Note that $\frac{\mathfrak{g}}{I}$ is still a free 2 -step nilpotent Lie algebra (on $n-1$ generators). We have established that in $\frac{\mathfrak{g}}{I}$ there are $2(k-1)$ vectors

$$
V_{1}^{\prime}+I, \ldots, V_{k-1}^{\prime}+I, W_{1}^{\prime}+I, \ldots, W_{k-1}^{\prime}+I
$$

for which the natural projections onto $\frac{\frac{\mathfrak{g}}{I}}{\left[\frac{\mathfrak{g}}{I}, \frac{\mathfrak{g}}{I}\right]}=\frac{\mathfrak{g}}{[\mathfrak{g}, \mathfrak{g}]+I}$ are linearly independent and such that

$$
\left[V_{1}^{\prime}, W_{1}^{\prime}\right]+\ldots+\left[V_{k-1}^{\prime}, W_{k-1}^{\prime}\right]+I=\left[\tilde{V}_{1}, \tilde{W}_{1}\right]+\ldots+\left[\tilde{V}_{k-2}, \tilde{W}_{k-2}\right]+I .
$$

This contradicts the truth of the lemma for $k-1$.

COROLlary 2.6. Over any field $K$ there are up to isomorphism exactly $\left\lfloor\frac{n}{2}\right\rfloor$ Lie algebras of type $T\left(n, \frac{(n+1)(n-2)}{2}\right)$.

Proof. Let $\mathfrak{g}$ be the free 2-step nilpotent Lie algebra on $n$ generators (i.e. the unique $T\left(n, \frac{n(n-1)}{2}\right)$ Lie algebra). Then, for each $k \in\left\{1,2, \ldots,\left\lfloor\frac{n}{2}\right\rfloor\right\}$ there is an element $X$ of length $k$ in $[\mathfrak{g}, \mathfrak{g}]$. (Choose $2 k$ linearly independent vectors in $\frac{\mathfrak{g}}{[\mathfrak{g}, \mathfrak{g}]}$ and form the corresponding sum of $k$ Lie brackets.) For each such element $X$ we obtain a Lie algebra $\frac{\mathfrak{g}}{\langle X\rangle}$ whose isomorphism type is determined completely by length $(X)$.

3. Hyperbolic automorphisms on $T\left(n, \frac{(n+1)(n-2)}{2}\right)$ Lie algebras. In [3, Lemma 5.1] it was shown that $T(3,2)$ groups and $T(4,5)$ groups do not admit any hyperbolic automorphism. In this section, we shall see that for any $n \geq 5$ there are $T\left(n, \frac{(n+1)(n-2)}{2}\right)$ groups admitting a hyperbolic automorphism. In fact, we shall characterize exactly when such a group admits a hyperbolic automorphism. Therefore we need some results on the Lie algebra level.

From now on $\mathfrak{h}$ will denote a rational Lie algebra which is obtained as a quotient of the form $\mathfrak{h}=\frac{\mathfrak{g}}{\langle X\rangle}$, where $\mathfrak{g}$ is a free 2-step nilpotent Lie algebra on $n$ generators over $\mathbb{Q}$ and $0 \neq X \in[\mathfrak{g}, \mathfrak{g}]$.

Any automorphism $\varphi \in \operatorname{Aut}(\mathfrak{h})$ is induced by a $\tilde{\varphi} \in \operatorname{Aut}(\mathfrak{g})$, with $\tilde{\varphi}(\langle X\rangle)=\langle X\rangle$; this means that

$$
\exists \lambda \in \mathbb{Q}_{0}: \tilde{\varphi}(X)=\lambda X .
$$

Let $\bar{\varphi}(=\overline{\tilde{\varphi}})$ denote the automorphism of $\frac{\mathfrak{h}}{[\mathfrak{h}, \mathfrak{h}]}\left(=\frac{\mathfrak{g}}{[\mathfrak{g}, \mathfrak{g}]}\right)$ induced by $\varphi($ resp. $\tilde{\varphi})$. Also let $\varphi_{0}$ (resp. $\tilde{\varphi}_{0}$ ) denote the restriction of $\varphi$ to $[\mathfrak{h}, \mathfrak{h}]$ (resp. of $\tilde{\varphi}$ to $[\mathfrak{g}, \mathfrak{g}]$ ).

Let $\lambda_{1}, \lambda_{2}, \ldots, \lambda_{n}$ denote the eigenvalues of $\bar{\varphi}$ (eigenvalues of multiplicity $>1$ are listed as many times as their multiplicity). In [4] (see also [3]) the following lemma was proved. 
LEMMA 3.1. With the notation above, the following properties hold.

1. The eigenvalues of $\tilde{\varphi}_{0}$ are $\lambda_{1} \lambda_{2}, \lambda_{1} \lambda_{3}, \ldots, \lambda_{1} \lambda_{n}, \lambda_{2} \lambda_{3}, \ldots, \lambda_{n-1} \lambda_{n}$.

2. If we denote the characteristic polynomial of a linear map $\psi$ by $p_{\psi}(x)$, then we have

$$
p_{\tilde{\varphi}}(x) \in \mathbb{Z}[x] \Longleftrightarrow p_{\varphi}(x) \in \mathbb{Z}[x] \Longleftrightarrow p_{\bar{\varphi}}(x) \in \mathbb{Z}[x] .
$$

Also if $p_{\tilde{\varphi}}(x) \in \mathbb{Z}[x]$, then

(a) $p_{\tilde{\varphi}}(x)$ has unit constant term $\Longleftrightarrow p_{\varphi}(x)$ has unit constant term

(b) $p_{\tilde{\varphi}_{0}}(x) \in \mathbb{Z}[x]$ and $p_{\varphi_{0}}(x) \in \mathbb{Z}[x]$.

$\Longleftrightarrow p_{\bar{\varphi}}(x)$ has unit constant term.

Having this information in mind, we can now prove the main result of our paper on the Lie algebra level.

THEOREM 3.2. Let $\mathfrak{g}$ be a free 2-step nilpotent Lie algebra over $\mathbb{Q}$ on $n$ generators and let $0 \neq X \in[\mathfrak{g}, \mathfrak{g}]$. Then $\mathfrak{h}=\frac{\mathfrak{g}}{\langle X\rangle}$ admits a hyperbolic automorphism whose characteristic polynomial has integer coefficients and with unit constant term if and only if $n \geq 5$ and length $(X)=1$.

Proof. First suppose that $\mathfrak{h}$ admits a hyperbolic automorphism $\varphi$ with $p_{\varphi}(x) \in \mathbb{Z}[x]$ and where $p_{\varphi}(x)$ has unit constant term. By [3, Lemma 5.1], we know that $n \geq 5$.

Let $\tilde{\varphi}, \bar{\varphi}, \lambda, \ldots$ have the same meaning as before. We claim that the eigenvalue $\lambda$ (belonging to the eigenvector $X$ for $\tilde{\varphi}$ ) is equal to \pm 1 . This follows from the fact that

$$
p_{\tilde{\varphi}}(x)=(x-\lambda) p_{\varphi}(x) .
$$

As $p_{\varphi}(x) \in \mathbb{Z}[x]$, the previous lemma implies that $p_{\tilde{\varphi}}(x) \in \mathbb{Z}[x]$ and therefore also $(x-\lambda) \in \mathbb{Z}[x]$. As both $p_{\tilde{\varphi}}(x)$ and $p_{\varphi}(x)$ have unit constant term, also $(x-\lambda)$ must have unit constant term, and thus $\lambda= \pm 1$.

By considering $\varphi^{2}$ instead of $\varphi$ (and thus $\tilde{\varphi}^{2}$ instead of $\tilde{\varphi}$ ), we can assume that $\lambda=1$. (Note that by the hyperbolicity of $\varphi, \lambda=1$ is an eigenvalue of multiplicity 1 .) Again, by the lemma above, we may assume that $\lambda=\lambda_{1} \lambda_{2}$.

Now, let $\mathfrak{g}_{\mathbb{C}}$ denote the complexification of $\mathfrak{g}$; i.e. $\mathfrak{g}_{\mathbb{C}}=\mathbb{C} \otimes \mathfrak{g}$ (tensor over $\mathbb{Q}$ ). There is a natural embedding of $\mathfrak{g}$ into $\mathfrak{g}_{\mathbb{C}}$ and we shall consider $\mathfrak{g}$ as a subset of $\mathfrak{g}_{\mathbb{C}}$. Let $X \in[\mathfrak{g}, \mathfrak{g}]$ of length $k$ (seen as an element of $[\mathfrak{g}, \mathfrak{g}]$ !). Then

$$
X=\left[V_{1}, W_{1}\right]+\ldots+\left[V_{k}, W_{k}\right] \text {, with } V_{1}, \ldots, W_{k} \in \mathfrak{g}
$$

such that the canonical projections $p\left(V_{1}\right), p\left(V_{2}\right), \ldots, p\left(W_{k}\right)$ in $\frac{\mathfrak{g}}{[\mathfrak{g}, \mathfrak{g}]}$ are linearly independent. But then, also the natural projections of $V_{1}, V_{2}, \ldots, W_{k}$ onto $\frac{\mathfrak{g}_{\mathbb{C}}}{\left[\mathfrak{g}_{\mathbb{C}}, \mathfrak{g}_{\mathrm{C}}\right]}$ are linearly independent, showing that length $(X)=k$, where now $X$ is seen as an element of $\left[\mathfrak{g}_{\mathbb{C}}, \mathfrak{g}_{\mathbb{C}}\right]$.

The automorphisms $\varphi, \tilde{\varphi}, \bar{\varphi}$ can all be considered on the complex level too. In $\mathfrak{g}_{\mathbb{C}}$, there exist an eigenvector $V$ (resp. $W$ ) corresponding to the eigenvalue $\lambda_{1}$ (resp. $\lambda_{2}$ ). Then

$$
\tilde{\varphi}[V, W]=[\tilde{\varphi}(V), \tilde{\varphi}(W)]=\lambda_{1} \lambda_{2}[V, W]=\lambda[V, W] .
$$

This implies that $X$ belongs to the space spanned by $[V, W]$ and therefore $X$ is of length 1 . 
Now, assume that length $(X)=1$ and $n \geq 5$. Consider the following matrices with determinant 1:

$$
\begin{aligned}
& A=\left(\begin{array}{ll}
2 & 1 \\
1 & 1
\end{array}\right), B_{1}=\left(\begin{array}{lll}
1 & 1 & 1 \\
1 & 2 & 2 \\
1 & 2 & 3
\end{array}\right), \\
& B_{2}=\left(\begin{array}{cccc}
0 & 1 & 0 & 0 \\
0 & 0 & 1 & 0 \\
0 & 0 & 0 & 1 \\
-1 & 6 & -1 & -5
\end{array}\right), \quad B_{3}=\left(\begin{array}{lllll}
1 & 1 & 1 & 1 & 1 \\
1 & 2 & 2 & 2 & 2 \\
1 & 2 & 3 & 3 & 3 \\
1 & 2 & 3 & 4 & 4 \\
1 & 2 & 3 & 4 & 5
\end{array}\right) .
\end{aligned}
$$

Let $\lambda_{1}, \lambda_{2}$ denote the eigenvalues of $A$ and let $\lambda_{3}, \lambda_{4}, \ldots, \lambda_{14}$ be the eigenvalues of $B_{1}, B_{2}$ and $B_{3}$. One computes that

$$
\lambda_{1} \lambda_{2}=1 \text { and }\left|\lambda_{i} \lambda_{j}\right| \neq 1 \text { if }\{i, j\} \neq\{1,2\}
$$

As $n \geq 5, n$ can be written as $n=i+4+3 k$ for some $i \in\{1,2,3\}$ and $k \in \mathbb{N}$. Consider the block diagonal matrix

$$
\left.C=\left(\begin{array}{c|c|c|c|c|c}
A & 0 & 0 & 0 & \ldots & 0 \\
\hline 0 & B_{i} & 0 & 0 & \ldots & 0 \\
\hline 0 & 0 & B_{1} & 0 & \ldots & 0 \\
\hline 0 & 0 & 0 & B_{1} & \ldots & 0 \\
\hline \vdots & \vdots & \vdots & \vdots & \ddots & \vdots \\
\hline 0 & 0 & 0 & 0 & \ldots & B_{1}
\end{array}\right)\right\} k \text { times } B_{1}
$$

This matrix can be seen as a matrix representation of a linear isomorphism $\bar{\varphi}$ of $\frac{\mathfrak{g}}{[\mathfrak{g}, \mathfrak{g}]}$. This isomorphism $\bar{\varphi}$ can be lifted to a Lie algebra automorphism $\tilde{\varphi}$ of $\mathfrak{g}$. By construction $\tilde{\varphi}$ has one eigenvalue equal to 1 (and of multiplicity 1). Let $Y \neq 0$ be an eigenvector corresponding to this eigenvalue. Then $Y$ is of length 1. (It is just the Lie bracket $\left[V_{1}, V_{2}\right]$, if $p\left(V_{1}\right), p\left(V_{2}\right), \ldots, p\left(V_{n}\right)$ is the basis of $\frac{\mathfrak{g}}{[\mathfrak{g}, \mathfrak{g}]}$, with respect to which $C$ is the matrix representation of $\bar{\varphi}$.) The induced automorphism on $\frac{\mathfrak{g}}{\langle Y\rangle}$ is a hyperbolic automorphism, whose characteristic polynomial has unit constant term and integer coefficients. As $\frac{\mathfrak{g}}{\langle X\rangle} \cong \frac{\mathfrak{g}}{\langle Y\rangle}$ the proof is finished.

4. Back to Anosov diffeomorphisms. We now interpret the result obtained in terms of Anosov diffeomorphisms on nilmanifolds. Theorem 3.2 can be rephrased as follows.

THEOREM 4.1. Let $M=N \backslash G$ be a 2-step nilmanifold, where $N$ is a $T\left(n, \frac{(n+1)(n-2)}{2}\right)$ group. Suppose that the Lie algebra $\mathfrak{g}_{\mathbb{Q}}$, corresponding to $N$, is written as a quotient of a free 2-step nilpotent Lie algebra by a 1-dimensional ideal $\langle X\rangle$. Then $M$ admits an Anosov diffeomorphism if and only if $n \geq 5$ and length $(X)=1$. 
It has been brought to our attention by S. G. Dani that one direction of the above theorem can be proved in a more general setting. To explain this, we need a little more notation.

Let $\mathfrak{g}$ be the real free 2-step nilpotent Lie algebra on $n$ generators $V_{1}, V_{2}, \ldots, V_{n}$. For each $k$ we define

$$
\mathfrak{h}_{n, k}=\frac{\mathfrak{g}}{I_{k}}, \text { with } I_{k}=\operatorname{span}\left\{\left[V_{p}, V_{q}\right] \mid 1 \leq p, q \leq k\right\}
$$

Let $H_{n, k}$ denote the connected and simply connected nilpotent Lie group corresponding to $\mathfrak{h}_{n, k}$.

THEOREM 4.2. Let $2 \leq k \leq n-3$. If $N$ is a lattice of the Lie group $H_{n, k}$, then the nilmanifold $N \backslash H_{n, k}$ admits an Anosov diffeomorphism.

Proof. By Theorem 2.2 of [2], it suffices to show that there exists a semisimple group of Lie algebra automorphisms of $\mathfrak{h}_{n, k}$ containing a hyperbolic element. We claim that we can take this semisimple group to be the group $S=S L_{k}(\mathbb{R}) \times S L_{n-k}(\mathbb{R})$. Indeed, this group $S$ acts on the vector space with basis $V_{1}, V_{2}, \ldots V_{n}$, in such a way that the $S L_{k}(\mathbb{R})$-part (resp. $S L_{n-k}(\mathbb{R})$-part) acts on the first $k$ (resp. last $n-k$ ) basis vectors. This action extends to an action on $\mathfrak{g}$, and $I_{k}$ is invariant under this action. It follows that the group $S$ acts on the Lie algebra $\mathfrak{h}_{n, k}$. It is easy to see that this action contains hyperbolic elements when $k \geq 2$ and $n-k \geq 3$, which proves the theorem.

Part of Theorem 4.1 is recovered from this theorem, by taking $k=2$ and $n \geq 5$.

The reader may wonder whether or not the criterion of Theorem 4.1 is useful in practice. We claim that it is and the rest of this paper is devoted to illustrate this claim.

Let $N$ be a torsion free, finitely generated, 2-step nilpotent group. Then $N$ has a presentation of the form

$$
\begin{gathered}
N=\left\langle a_{1}, a_{2}, \ldots, a_{n}, b_{1}, b_{2}, \ldots, b_{k}\right|\left[a_{i}, a_{j}\right]=b_{1}^{\alpha_{i, j, 1}} b_{2}^{\alpha_{i, j, 2}} \ldots b_{k}^{\alpha_{i, j, k}} \quad(1 \leq i<j \leq n) \\
{\left[a_{i}, b_{j}\right]=1 \quad(1 \leq i \leq n, \quad 1 \leq j \leq k)} \\
\left.\left[b_{i}, b_{j}\right]=1 \quad(1 \leq i<j \leq k)\right\rangle .
\end{gathered}
$$

It is then an easy application of the Campbell-Baker-Hausdorff formula to see that

$$
\begin{array}{r}
\mathfrak{g}_{\mathbb{Q}}=\left\langle A_{1}, A_{2}, \ldots, A_{n}, B_{1}, B_{2}, \ldots, B_{k}\right|\left[A_{i}, A_{j}\right]=\sum_{l=1}^{k} \alpha_{i, j, l} B_{l} \quad(1 \leq i<j \leq n) \\
{\left[A_{i}, B_{j}\right]=0 \quad(1 \leq i \leq n, \quad 1 \leq j \leq k)} \\
\left.\left[B_{i}, B_{j}\right]=0 \quad(1 \leq i<j \leq k)\right\rangle
\end{array}
$$

so it is really easy to change from the group to the Lie algebra level.

Note that a presentation like the above suggests that $\mathfrak{g}_{\mathbb{Q}}$ is of type $T(n, k)$. This is indeed true if $\left[\mathfrak{g}_{\mathbb{Q}}, \mathfrak{g}_{\mathbb{Q}}\right]=\left\langle B_{1}, B_{2}, \ldots, B_{k}\right\rangle$. As $\left[\mathfrak{g}_{\mathbb{Q}}, \mathfrak{g}_{\mathbb{Q}}\right]$ is spanned by the brackets $\left[A_{i}, A_{j}\right]$, we must check whether or not the space spanned by the vectors $\sum_{l=1}^{k} \alpha_{i, j, l} B_{l}$ $(1 \leq i<j \leq n)$ is $k$-dimensional. This comes down to checking whether or not

$$
\operatorname{rank}\left(\begin{array}{cccc}
\alpha_{1,2,1} & \alpha_{1,2,2} & \ldots & \alpha_{1,2, k} \\
\alpha_{1,3,1} & \alpha_{1,3,2} & \ldots & \alpha_{1,3, k} \\
\vdots & \vdots & \ddots & \vdots \\
\alpha_{n-1, n, 1} & \alpha_{n-1, n, 2} & \ldots & \alpha_{n-1, n, k}
\end{array}\right)=k
$$


Now, assume that $k=\frac{(n+1)(n-2)}{2}$. We know that $N\left(\mathfrak{g}_{\mathbb{Q}}\right)$ is actually a $T(n, k)$ group $\left(T(n, k)\right.$ Lie algebra) and so $\mathfrak{g}_{\mathbb{Q}}$ can be written as a quotient of a free 2-step nilpotent Lie algebra by a 1-dimensional ideal $\langle X\rangle$. We need to know $X$.

To see how this works, let $\mathfrak{h}$ be the rational free 2-step nilpotent Lie algebra on $n$ generators $C_{1}, C_{2}, \ldots, C_{n}$, so that $\mathfrak{h}$ has a vector space basis

$$
C_{1}, C_{2}, \ldots, C_{n},\left[C_{1}, C_{2}\right],\left[C_{1}, C_{3}\right], \ldots,\left[C_{n-1}, C_{n}\right]
$$

and there exists a Lie algebra morphism

$$
\psi: \mathfrak{h} \rightarrow \mathfrak{g}_{\mathbb{Q}}, \text { with } \psi\left(C_{i}\right)=A_{i} \text { and } \operatorname{kernel}(\psi)=\langle X\rangle
$$

Therefore, we need to know a non-zero $X \in[\mathfrak{h}, \mathfrak{h}]$ with $\psi(X)=0$. In other words we search for a non-zero linear combination

$$
X=\beta_{1,2}\left[C_{1}, C_{2}\right]+\beta_{1,3}\left[C_{1}, C_{3}\right]+\cdots+\beta_{n-1, n}\left[C_{n-1}, C_{n}\right] \text { with } \psi(X)=0 .
$$

As $\psi\left(\left[C_{i}, C_{j}\right]\right)=\left[A_{i}, A_{j}\right]$, this is the same as looking for a set of $\beta_{i, j}$, which are not all zero and such that

$$
\begin{aligned}
\beta_{1,2}\left(\alpha_{1,2,1}, \alpha_{1,2,2}, \ldots, \alpha_{1,2, k}\right) & + \\
\beta_{1,3}\left(\alpha_{1,3,1}, \alpha_{1,3,2}, \ldots, \alpha_{1,3, k}\right) & + \\
\vdots & \vdots \\
\beta_{n-1, n}\left(\alpha_{n-1, n, 1}, \alpha_{n-1, n, 2}, \ldots, \alpha_{n-1, n, k}\right) & =(0,0, \ldots, 0) .
\end{aligned}
$$

Hence the problem is reduced to finding a solution to a linear system of equations, which is a trivial linear algebra problem. Once this solution is found, it remains to find out if the length of $X=\beta_{1,2}\left[C_{1}, C_{2}\right]+\beta_{1,3}\left[C_{1}, C_{3}\right]+\cdots+\beta_{n-1, n}\left[C_{n-1}, C_{n}\right]$ is equal to 1 or not. The length of $X$ can be obtained by using iteratively and as long as possible the process in the proof of Lemma 2.2 to write $X$ as a sum of fewer Lie brackets. The moment this becomes impossible, we have found the length of $X$.

Let us illustrate the above remarks by means of a concrete example. Let $N$ be a 2-step nilpotent group, such that the corresponding $\mathfrak{g}_{\mathbb{Q}}$ is given by

$$
\begin{aligned}
& \mathfrak{g}_{\mathbb{Q}}=\langle A_{1}, A_{2}, A_{3}, A_{4}, A_{5}, B_{1}, B_{2}, B_{3}, B_{4}, B_{5}, B_{6}, B_{7}, B_{8}, B_{9} \mid \\
& {\left[A_{i}, B_{j}\right]=0(1 \leq i \leq 5),(1 \leq j \leq 9) } \\
& {\left[B_{i}, B_{j}\right]=0 \quad(1 \leq i<j \leq 9) } \\
& {\left[A_{1}, A_{2}\right]=B_{1} } \\
& {\left[A_{1}, A_{3}\right]=B_{2} } \\
& {\left[A_{1}, A_{4}\right]=B_{3} } \\
& {\left[A_{1}, A_{5}\right]=B_{4} } \\
& {\left[A_{2}, A_{3}\right]=B_{5}+2 B_{6} } \\
& {\left[A_{2}, A_{4}\right]=B_{4}+B_{5}+B_{6} } \\
& {\left[A_{2}, A_{5}\right]=B_{7}+3 B_{8}+B_{9} } \\
& {\left[A_{3}, A_{4}\right]=B_{8}+5 B_{9} } \\
& {\left[A_{3}, A_{5}\right]=B_{9} } \\
& {\left.\left[A_{4}, A_{5}\right]=-2 B_{1}-B_{2}-7 B_{8}-35 B_{9}\right\rangle }
\end{aligned}
$$


The matrix consisting of the parameters $\alpha_{i, j, l}$ is of the form

$$
\left(\begin{array}{ccccccccc}
1 & 0 & 0 & 0 & 0 & 0 & 0 & 0 & 0 \\
0 & 1 & 0 & 0 & 0 & 0 & 0 & 0 & 0 \\
0 & 0 & 1 & 0 & 0 & 0 & 0 & 0 & 0 \\
0 & 0 & 0 & 1 & 0 & 0 & 0 & 0 & 0 \\
0 & 0 & 0 & 0 & 1 & 2 & 0 & 0 & 0 \\
0 & 0 & 0 & 1 & 1 & 1 & 0 & 0 & 0 \\
0 & 0 & 0 & 0 & 0 & 0 & 1 & 3 & 1 \\
0 & 0 & 0 & 0 & 0 & 0 & 0 & 1 & 5 \\
0 & 0 & 0 & 0 & 0 & 0 & 0 & 0 & 1 \\
-2 & -1 & 0 & 0 & 0 & 0 & 0 & -7 & -35
\end{array}\right)
$$

for which it is easy to see that it is of rank 9 , so that the Lie algebra is a $T(5,9)$ Lie algebra. To find the corresponding $X=\sum \beta_{i, j}\left[C_{i}, C_{j}\right]$ in the free 2-step nilpotent Lie algebra on 5 generators, we must find a non-zero solution of the system of linear equations

$$
\begin{gathered}
\beta_{1,2}(1,0,0,0,0,0,0,0,0)+ \\
\beta_{1,3}(0,1,0,0,0,0,0,0,0)+ \\
\beta_{1,4}(0,0,1,0,0,0,0,0,0)+ \\
\beta_{1,5}(0,0,0,1,0,0,0,0,0)+ \\
\beta_{2,3}(0,0,0,0,1,2,0,0,0)+ \\
\beta_{2,4}(0,0,0,1,1,1,0,0,0)+ \\
\beta_{2,5}(0,0,0,0,0,0,1,3,1)+ \\
\beta_{3,4}(0,0,0,0,0,0,0,1,5)+ \\
\beta_{3,5}(0,0,0,0,0,0,0,0,1)+ \\
\beta_{4,5}(-2,-1,0,0,0,0,0,-7,-35)=(0,0, \ldots, 0) .
\end{gathered}
$$

One non-zero solution is given by $\beta_{1,2}=2, \beta_{1,3}=1, \beta_{3,4}=7, \beta_{4,5}=1$ and $\beta_{i, j}=0$ in all other cases. Thus

$$
\begin{aligned}
X & =2\left[C_{1}, C_{2}\right]+\left[C_{1}, C_{3}\right]+7\left[C_{3}, C_{4}\right]+\left[C_{4}, C_{5}\right] \\
& =\left[C_{1}, 2 C_{2}+C_{3}\right]+\left[C_{4},-7 C_{3}+C 5\right] .
\end{aligned}
$$

This last expression cannot be reduced any further (by Lemma 2.5) and therefore length $(X)=2$. We conclude that $N$ does not admit a hyperbolic automorphism and that the corresponding nilmanifold $N \backslash G$ does not admit an Anosov diffeomorphism.

\section{REFERENCES}

1. C. Cassidy, N. Kennedy and D. Scevenels, Hyperbolic automorphisms for groups in $\mathrm{T}(4,2)$, Contemp Math. 262 (2000), 171-175.

2. S. Dani, Nilmanifolds with Anosov automorphisms, J. London Math. Soc. (2) 18 (1978), 553-559.

3. K. Dekimpe, Hyperbolic automorphisms and Anosov diffeomorphisms on nilmanifolds, Trans. Amer. Math. Soc. 353 (2001), 2859-2877. 
4. K. Dekimpe and W. Malfait, A special class of nilmanifolds admitting an Anosov diffeomorphism, Proc. Amer. Math. Soc. 128 (2000), 2171-2179.

5. A. I. Mal'cev, On a class of homogeneous spaces, Translations Amer. Math. Soc. 39 (1951), 1-33.

6. W. Malfait, Anosov diffeomorphisms on nilmanifolds of dimension at most six, Geom Dedicata 79 (2000), 291-298.

7. A. Manning, There are no new Anosov diffeomorphisms on tori, Amer. J. Math. 96 (1974), 422-429.

8. H. L. Porteous, Anosov diffeomorphisms of flat manifolds, Topology 11 (1972), 307-315.

9. M. S. Raghunathan, Discrete subgroups of Lie groups (Springer-Verlag, 1972).

10. D. Segal, Polycyclic groups (Cambridge University Press, 1983). 